\title{
The evolution of wellbeing in educational policy in Ireland: Towards an interdisciplinary approach
}

\author{
Margaret Nohilly · Fionnuala Tynan
}

\begin{abstract}
This paper examines how wellbeing has evolved in educational policy over the last decade in Ireland. The influence of the developments in national policy on wellbeing are also considered. While the concept of wellbeing is progressing in educational policy documents and becoming embedded in the language of the school community, a review of literature in the area highlights that it is difficult to define and to understand. Although wellbeing is a central theme in Early Years education and an area of learning in post-primary education for Junior Cycle in the Irish context, presently it can be regarded as implicit in the primary school curriculum rather than a defined theme or area of learning. The Draft Curriculum Framework published by the National Council for Curriculum and Assessment (NCCA) in February 2020 includes wellbeing as one of five broad curriculum areas. This paper presents a critical reflection on the inclusion of wellbeing as an area of learning in a redeveloped primary school curriculum.
\end{abstract}

Keywords: wellbeing; curriculum redevelopment; primary school; educational policy; Ireland

\section{Introduction}

In 1999 when the current iteration of the Irish primary school curriculum was launched, its overall aims included developing each child's potential to the full, encouraging a love of learning and helping children develop skills that they would use throughout their lives (Government of Ireland 1999a). It may well be argued that the wellbeing of each child was a central tenet of this curriculum, reflected in its vision to "nurture the child in all dimensions of his or her life-spiritual, moral, cognitive, emotional, imaginative, aesthetic, social and physical" (Government of Ireland 1999a, p.6). In the subject area of Social, Personal and Health Education (SPHE), the wellbeing of the child is referenced, but it is not a term that is explicitly used throughout or indeed across the curriculum. Twenty years later as the National Council for Curriculum and Assessment (NCCA) presents the Draft Primary Curriculum Framework for consultation, the wellbeing of each child is reflected in the key competencies and in the five broad curriculum areas proposed (NCCA 2020). This reflects developments in wellbeing, not only in education, but in national policy in the intervening time. It also ensures that wellbeing is a central theme across all stages of education; from early childhood through to post-primary. While educators may be in agreement that the wellbeing of each child must be a paramount consideration, what exactly do we mean when we talk about wellbeing?

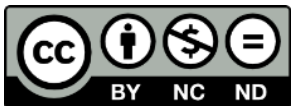

Copyright belongs to the author(s) www.internationaljournalofwellbeing.org 


\subsection{What is wellbeing?}

Dodge et al. (2012) consider that wellbeing is the point of balance between the resources one has and the challenges one faces. This perspective, while worthy of reflection, does not adequately define wellbeing in a comprehensive way. Indeed, defining wellbeing is not straightforward. In the first instance it is complicated by variations of its spelling (wellbeing, well-being, well being). This is reflected in Irish educational policy documents. In the wellbeing guidelines for postprimary and primary schools published jointly by the Department of Education and Skills (DES) and the Department of Health (DoH) in 2013 and 2015 respectively, the spelling is well-being, while the Wellbeing Policy Statement and Framework for Practice published by DES in 2018 refers to 'wellbeing' throughout. Wellbeing is a very broad concept and encompasses many subsets such as emotional wellbeing, social wellbeing, psychological wellbeing and mental health and wellbeing (Svane et al. 2019). It is further complicated by the fact that it is a multidisciplinary concept applied across various fields from economics to politics, from counselling to education and across psychology and philosophy. In fact, following a systematic review of wellbeing literature, Svane et al. (2019) concur with Bache et al. (2015) that defining wellbeing is a 'wicked' problem given the multidimensional nature of the term and the many different definitions that exist. According to Souttera, O'Steena and Gilmore (2014, p.497) "despite significant attention to conceptualising and evaluating well-being in academic and policy circles, well-being remains a narrowly defined, if not undefined, term in education, complicating efforts to plan for and monitor it effectively". Gillet-Swan and Sergeant (2015, p.146) conclude that "wellbeing is a highly popularised and aspirational concept in philosophical, psychological, medical and health literature". Despite this, "there has not been a clearly defined and workable definition of wellbeing that incorporates its multifaceted, multidimensional and progressive elements". In the Irish educational context, the development of wellbeing has been influenced by national policy developments and educational developments in other countries.

\subsection{Influences on the development of wellbeing in education}

The development of policy in relation to wellbeing in Ireland is not just a matter for education, it is primary concern for the DoH also and indeed for all Government Departments who work with and support children. Significantly, the first National Children's Strategy was published in Ireland in 2000 and in many ways it provided an important blueprint for improving children's lives, proposing a vision where

children are respected as young citizens with a valued contribution to make and a voice of their own; where all children are cherished and supported by family and the wider society; where they enjoy a fulfilling childhood and realise their potential (DES and DoH, 2000).

One of the significant outcomes of this initial strategy was the establishment of a national longitudinal study of children in Ireland and as a result, the Growing Up in Ireland study was launched. This government-funded study of children started in 2006 and follows the progress of 8,000 nine-year olds and 10,000 nine-month-olds. While the primary aim of the study is to inform government policy in relation to children and their families, the study objectives seek to examine the progress and wellbeing of children at critical periods from birth to adulthood. Key findings from the Cohort '08 (the Infant cohort) who were last interviewed at age nine indicate that most nine-year olds are doing well in terms of their socio-emotional and behavioural wellbeing (growingup.ie). This shows that national policy is being realised, despite the lack of definition in relation to wellbeing. 
Better Outcomes, Brighter Futures: The National Policy Framework for Children and Young People 2014-2020 is the first national policy framework in Ireland for children and young people and establishes a shared set of outcomes which all governments departments work towards. The first of five outcomes listed outlines that children will be active and healthy, with positive physical and mental wellbeing (Government of Ireland 2014). While the inclusion of wellbeing in the five outcomes highlights its priority, again there is a particular focus on mental wellbeing. There is a similar focus identified in the Healthy Ireland framework developed by the $\mathrm{DoH}$ in 2013. This framework establishes a vision where "everyone can enjoy physical and mental health and wellbeing to their full potential, where wellbeing is valued and supported at every level of society and is everyone's responsibility" (DoH 2013, p.6). These policies confirm a cross-governmental approach to improve all aspects of health and wellbeing for children and young people.

Developments in wellbeing are also taking place in the Irish educational context. They have been influenced by international educational developments, in particular those in Scotland. Wellbeing in Scotland is described in terms of eight indicators; every child and young person should be safe, healthy, achieving, nurtured, active, respected, responsible and included (https://www.gov.scot/policies/girfec/wellbeing-indicators-shanarri/). These indicators are adapted for use in the Irish context with wellbeing indicators included in the post-primary guidelines (NCCA 2017). The post-primary guidelines were updated in 2021 and the wellbeing indicators are included in the guidelines, with relevant descriptors for each indicator (NCCA 2021). The status and place of wellbeing across curricula in other countries varies also. Health and wellbeing education is part of the mandatory or state curriculum in many countries including Australia, Finland, Denmark and New Zealand. In Scotland, personal wellbeing occupies a more prominent curriculum role and is, along with literacy and numeracy, one of three key responsibilities of all teachers, and a subject specific responsibility for those teachers who have a specific health and wellbeing remit (Thornburn 2015). In other countries, health and wellbeing education finds itself vying for a place in official curriculum structures and is relegated to the status of non-statutory (Leahy and Simovska 2017). Indeed, arguments are well documented about the rise of 'therapeutic' education in the context of wellbeing education (Young 2013). These arguments contend that therapeutic education is turning children and young people into anxious and self-preoccupied individuals rather than aspiring, optimistic and resilient learners. While Beck (2012) argues that there is a dearth of reliable evidence in relation to this claim, there clearly has been a rise in social and emotional learning programmes in schools. Barry et al (2017) have shown that the integration and sustainability of such programmes within the context of whole-school systems is far from clearly established. Indeed, O Toole (2017, p.453) considers that wellbeing is an area that straddles the disciplinary fields of education, psychology and health and as a result there is a "pressing need to scrutinise current approaches in schools and to develop conceptual models that resonate with researchers and practitioners across the disciplinary boundaries". Developing a conceptual model from the interdisciplinary fields would be most useful in considering the place of wellbeing in the next iteration of the primary school curriculum.

\section{Educational and curriculum policy developments relating to wellbeing}

This section will pick up on some of the above developments in the Irish context and trace, in a chronological manner, the development of education and curriculum policy with regard to wellbeing. It is interesting that wellbeing was first introduced to the early years sector, then to the Junior Cycle programme and then to primary education. It is not yet addressed at Senior Cycle. 


\subsection{Early childhood}

While the place of wellbeing can be described as implicit within the current Irish primary school curriculum, the initial inclusion of wellbeing in Irish education dates back to 2009 when Aistear, the Early Childhood Curriculum Framework for children from birth to six years was published. The framework uses four interconnected themes to describe the content of children's learning and development: Wellbeing, Identity and Belonging, Communicating and Exploring and Thinking (NCCA 2009). Wellbeing, in this context, is outlined as having two major components: physical and psychological. The Aistear framework is experienced by many pupils in the primary school context, particularly in the infant classes (age four to six years). In practice, classrooms are often divided into interest areas such as; a construction area, a pretend play area, a quiet area and a messy play area and children engage in learning through a range of activities often linked to a particular theme. It is intended that these experiences will support the development of the children's physical and psychological wellbeing.

\subsection{Post-primary education}

In terms of wellbeing at post-primary education, the Well-Being in Post-Primary Schools: Guidelines for Mental Health Promotion and Suicide Prevention were published in 2013. The purpose of these guidelines was to support schools in developing a whole-school approach to mental health promotion and suicide prevention (DES and DoH 2013). The overarching focus of the guidelines is on a whole-school approach based on the continuum of support proposed by the National Educational Psychological Service, with which schools are familiar. Although all schools received a copy of the guidelines, given that there was no specific circular from the DES at the time alerting schools to their importance or no continuous professional development opportunities whereby teachers could engage with their content, the guidelines may have had limited impact in schools.

In 2015, the framework for junior cycle in post-primary was published. Wellbeing is one of the principles that underpins junior cycle education. Irish students now undertake learning in a new area entitled 'wellbeing' throughout the three years of junior cycle. The associated curriculum guidelines were published in 2017 and revised in 2021. It is interesting to note the change in spelling from the DES at this point, from 'well-being' to 'wellbeing'. This highlights the evolving nature of the concept. While the guidelines outline that teachers in all subjects can support student learning in the area of wellbeing, there is "a strong emphasis on the role that Civic, Social and Political Education (CSPE), Physical Education (PE) and Social, Personal and Health Education (SPHE) can play in supporting learning about wellbeing and learning for wellbeing" (NCCA 2017, p.46). The place of wellbeing in senior cycle education is at the discretion of the school as no formal guidelines for its implementation are developed. This is a clear gap in provision and fails to seize the opportunity that wellbeing education could provide for students as they engage in a highly pressurised examination system and prepare to embark on a significant transition from school and home life to that of work or further education.

\subsection{Primary Education}

While wellbeing is not an area of learning, or a 'theme' in primary school education, it is certainly a term that has become part of the language of the primary school context in recent years. In terms of curriculum at primary level, the subject area of SPHE, introduced in 1999, has always promoted the wellbeing of students. 
"Social, personal and health education provides particular opportunities to foster the personal development, health and well-being of the child and to help him/her to create and maintain supportive relationships and become an active and responsible citizen in society" (Government of Ireland 1999b, p.2).

The current SPHE curriculum supports many aspects of wellbeing, including helping children to develop both intrapersonal and inter-personal skills and to establish and maintain healthy patterns of behaviour. Furthermore, discrete areas are addressed that relate to wellbeing and include; self-identity and self-esteem, relationships and sexuality education, substance use, friendship and bullying, personal safety, decision making and feelings and emotions. SPHE has a very limited amount of curriculum time with just thirty minutes per week, the least amount of time given to any curricular area. Furthermore, given that it is twenty years since the curriculum was published, teachers do not seem to consider its real relevance in the current wellbeing space and tend to consider that wellbeing is something additional to SPHE. As with post-primary, WellBeing in Primary Schools: Guidelines for Mental Health Promotion were published in 2015 (DES and $\mathrm{DoH}$ 2015). Although the title has no reference to suicide prevention, the content of the guidelines is based on the post-primary school version. In a study undertaken with Irish primary school teachers on wellbeing, it emerged that many of the teachers were not aware that the guidelines were published and available in schools (Nohilly and Tynan 2019).

In 2018 the Wellbeing Policy Statement and Framework for Practice (2018-2023) was published by the DES, and is relevant for all schools and centres of education. In his foreword, the then Minister for Education, Joe McHugh, outlined that he had "set as a target that all schools and centres for education will have embedded a Self-Evaluation Wellbeing Promotion Process by 2023" (DES 2018, p.1). This framework therefore will become an important guideline for primary schools in the more formal development of wellbeing practices in schools. The framework promotes a whole-school approach to wellbeing through the four key areas of Culture and Environment, Curriculum, Policy and Planning and Relationships and Partnerships. These four areas are informed by the Schools for Health Process, an initiative of the Health Service Executive (HSE). School Self-Evaluation (SSE) is the vehicle by which the process will become embedded in schools. The requirement to use SSE to manage change within an educational context is not new. Since 1999, the School Development Planning Support initiative has been introduced to schools, promoting processes of reflection, use of evidence and action planning. In 2012, following the launch of the Literacy and Numeracy for Learning and Life, 2011-2020 strategy, SSE was introduced to schools and schools have since engaged with the process in an effort to improve standards in literacy, numeracy and other areas of teaching and learning. The wellbeing framework requires that schools "commit to a process of self-reflection to identify existing strengths, opportunities and challenges in their practice and incorporate a Wellbeing Promotion Process into their existing planning and self-evaluation processes" (DES 2018, p.24). Although the DES have established a dedicated SSE website for primary and post-primary schools and have delivered a number of important updates to schools via Circulars and 'SSE Update' newsletters, the requirements for schools in the area of wellbeing have not been clearly outlined, resulting in a lack of awareness from the schools' perspective in relation to requirements. Furthermore, the framework was published in July 2018, when schools were closed for the summer break. Although wellbeing will be a key priority for schools following the impact of the Coronavirus pandemic, many teachers are unaware of the requirements of the framework.

The recently published Draft Primary Curriculum Framework for Irish primary schools proposes that wellbeing will be included as a curriculum area in the next iteration of curriculum. The framework is due to be finalised and published in 2021, and the development of curriculum 
area specifications is proposed to be completed by 2024. The draft framework proposes a set of seven key competencies that are closely linked to both the Aistear and the Junior Cycle framework and 'fostering wellbeing' is one of the seven key competencies outlined. Wellbeing then, is one of five broad curriculum areas proposed for the redeveloped curriculum and incorporates the subject areas of Social, Personal and Values Education and Physical and Health Education. Of particular note is the increased time that is being considered for the area; two and a half hours per week at the Infant level and three hours per week from first to sixth class (NCCA 2020). While the proposed inclusion of wellbeing in the redeveloped curriculum is most welcome as is the increased time, the content and changes to the structure of the current SPHE and PE curricula will take particular negotiation and consideration.

\section{Definitions of wellbeing in education policy}

In the field of education, there are different interpretations of wellbeing and again, wellbeing and mental health are presented as synonymous in some documents (DES and DoH; 2013; 2015). Indeed, the terms are often used interchangeable and unsurprisingly, are also interlinked in research (Svane et al. 2019). In recent years, the discourse in relation to mental health has dominated, particularly in the context of school life. My World Survey 2, the National Study of Youth Mental Health in Ireland, presents a second wave of data on youth mental health in Ireland and highlights that levels of depression and anxiety have increased in adolescents and young adults (12-19 years) since the first survey was completed in 2012. Furthermore, levels of self-esteem, optimism and resilience; key protective factors in supporting mental health have decreased (Dooley et al. 2019). Despite the concerning growth in rates of anxiety among young people, a focus on mental health alone narrows the concept of wellbeing, particularly in the school context.

Many definitions of wellbeing are presented in educational policy documents (DES and DOH 2015; NCCA 2017 and 2021; DES 2018). Furthermore, a 'wellbeing working group', consisting of representation from the Teaching Council and Irish Primary Principals' Network propose a definition of wellbeing for schools (Authors). As the literature has highlighted, wellbeing is a 'wicked' problem and difficult to define, however a definition in the school context enables a common understanding across the whole school community. The definitions share the importance of the students' experience at school, including quality teaching and learning and a sense of belonging to the school community. The development of a conceptual framework to contextualise wellbeing would certainly support a broader understanding in the area and situate wellbeing within an educational framework. The multiple interpretations presented in the field of education has the potential to cause further confusion of an already complicated concept, or alternatively, it may offer schools choice in selecting a definition that is suitable to the context of the school. Given the requirement for schools to engage with the DES Wellbeing Policy Statement and Framework for Practice (2018), the definition of wellbeing presented therein is likely to be accepted by many schools. Specifically, the DES definition considers that

wellbeing is present when a person realises their potential, is resilient in dealing with the normal stresses of their life, takes care of their physical wellbeing and has a sense of purpose, connection and belonging to a wider community. It is a fluid way of being and needs nurturing throughout life (DES 2018, p.10).

The concept of wellbeing as fluid takes account of the multiplicity of factors that impact on wellbeing and furthermore of the potential for a state of wellbeing to be influenced by a range of external factors. It is important to consider wellbeing as a continuum with consideration of illbeing as part of the reality of human development. Simply put, illbeing represents a lack of 
wellbeing (O'Brien and O'Shea 2017). When illbeing is considered within a normative context, the concept can be viewed negatively as a state of illbeing such as negative moods, thoughts or feelings can be seen as a contradiction to wellbeing. However, as disruptions and setbacks are an integral part of wellbeing achievement, illbeing is therefore part of wellbing. Teachers and students have knowledge and experience of illbeing as part of the reality of human development and there is space for this experience to be included in an exploration of wellbeing. If it is ignored, students may interpret wellbeing as a solely positive state and ignore the circumstances where wellbeing struggles to flourish and be meaningful in practice. Illbeing is part of the human experience and can be seen as "crucial for human flourishing" (O'Brien and O'Shea 2017, p.30). While the place of wellbeing in the curriculum is so important, and particularly welcome in the primary school context, pupil experiences of wellbeing at school extends far beyond the curriculum space.

\section{The influence of schools on the wellbeing of pupils}

It is widely accepted that schools are settings that can have a great impact on the health and wellbeing of young people, given the influence they have on children at such an important stage in their lives (Deschesnes et al. 2014). Wellbeing has gained a prominent position on the educational landscape due to the wellbeing-learning connection. Wellbeing supports young people's cognitive and emotional engagement with school which influences educational achievement (Pietarinen, Soini and Phyalto 2014). Considering that pupils spend the majority of their school day in the classroom, "the day-to-day experience of teaching and learning within the classroom probably provides the greatest opportunity to contribute to student wellbeing" (NCCA 2017, p.32). Teachers, in their everyday teaching support the wellbeing of pupils through the implementation of the Aistear curriculum, the use of varied active methodologies and the implementation of a broad and balanced curriculum (Tynan and Nohilly 2018). When pupils have rich and engaging experiences of teaching and learning, across the curriculum, this is a key contributing factor to wellbeing in school. Indeed, one of the key successes of the 1999 Primary School Curriculum has been teachers' increased use of active learning methodologies, while one of the major challenges relates to curriculum overload (NCCA 2020). Reviewing and redeveloping the curriculum provides an important and timely opportunity to address curriculum overload, whilst ensuring pupils continue to experience rich and engaging learning experiences.

The school culture and climate is integral to wellbeing in schools. School culture and climate is difficult to define because much of it is unspoken and taken for granted (Tynan and Nohilly 2018). School culture is concerned with the norms and expectations that exist within an organisation. These shared views and beliefs may result from underlying individual values and assumptions and exert a powerful influence on psychological wellbeing and much of school culture is not very explicit but exists in the hidden curriculum of the school. Climate refers to how a school's environment affects an individual's sense of psychological safety, and the degree to which the environment promotes a sense of accomplishment and competence (Dessel 2010). The SPHE Curriculum advocates that a positive school culture and climate fosters the wellbeing of all members of the school and reflects an environment where everyone feels safe and experiences a sense of belonging (Government of Ireland 1999b). The Junior Cycle Wellbeing Guidelines (NCCA 2017), informed by the work of O'Brien and O' Shea (2017), highlight the central role that schools play in pupils learning about wellbeing and learning for wellbeing. Accordingly, learning about wellbeing occurs through specific areas of the curriculum and through wellbeing events that develop awareness, knowledge and skills about wellbeing. 
Learning for wellbeing occurs when pupils' whole experiences of school life, including the dayto-day interactions, both within and beyond the classroom, are respectful and caring. Learning for wellbeing takes account of the importance of school culture and climate. The for wellbeing is as important as the about wellbeing, but because the about wellbeing relates to curriculum and wellbeing events, it tends to dominate the wellbeing space.

Each individual classroom environment within a school has its own unique climate and is the space occupied by primary school pupils for the majority of the school day. The classroom climate impacts significantly on the quality of learning, pupil motivation and pupil engagement in classroom tasks. Teachers create the 'atmosphere' in the classroom through the attitude displayed to students, the classroom rules created, the interactions with pupils and the language used in the classroom (Tynan and Nohilly 2018). Pupils contribute to the classroom environment in a significant way also and should be encouraged to support the development of classroom rules and given responsibilities. The teacher-pupil relationship is a significant factor in pupil wellbeing. Positive relationships with teachers and a feeling of safety are important for pupil wellbeing within schools (Jamal et al. 2013). Pupils having a positive relationship with their teachers may contribute to school connectedness, which is identified as an environment where pupils believe adults in the school care about their learning and about them as individuals (Blum et al. 2004). A large body of international evidence shows an association between the quality of relationships between pupils and teachers and a number of pupil outcomes including; socioemotional wellbeing, engagement in schoolwork, feeling a sense of belonging in school, disciplinary problems and academic achievement (NCCA 2017). Learning for wellbeing will be significantly influenced by the classroom climate and in turn will support pupils learning about wellbeing.

The wellbeing of teachers and all school staff cannot be ignored. High levels of teacher wellbeing have been shown to have significant positive effect across a number of domains including health, life and chosen occupation (Turner and Theilking 2019). Weare (2015) considers that wellbeing in schools starts with staff. If teachers feel uncared for and burnt out themselves, it is hard for them to be genuinely motivated to promote the wellbeing of others. Part of the task of putting wellbeing on the school map involves creating opportunities for staff to consider their own wellbeing (NCCA 2017). Support for the wellbeing of staff is acknowledged in the DES Wellbeing Policy Statement and Framezork for Practice. In addition to building staff capacity to cope with challenge and adapt to change, it sustains engagement, enthusiasm and ability to model resilience (DES 2018).

\section{Considerations for wellbeing in a redeveloped primary curriculum}

Undoubtedly, wellbeing has taken its place on the national agenda in Ireland. This is evident in terms of national policy development and consequently, in terms of education policy developments. The inclusion of wellbeing as a key competency and as a broad curriculum area in the Draft Primary Curriculum Framework is very welcome and will ensure continuity from early childhood experiences through to Junior Cycle of post-primary education. However, given the ambiguity that surrounds the term, coupled with the fact that wellbeing is so much more than a curriculum experience for pupils, wellbeing in a redeveloped primary school curriculum needs to be understood and organised so that is takes account of the holistic school experience of pupils.

Even though it is over a decade since the Aistear framework was launched, no systematic evaluation of the implementation of the framework in the infant classes in Ireland has been undertaken (Smyth 2012). Consequently, it is difficult to determine how wellbeing is integrated into children's learning at this stage. Continuous professional development of the 
implementation of Aistear was not made available by the DES to all teachers, and this has impacted on implementation also. Wellbeing as an area of learning in Junior Cycle is also in its infancy. An exploratory study completed as part of a Master's thesis on the impact of the involvement of teachers of SPHE, Civic, Social and Political Education (CSPE) and PE in the new wellbeing programme at Junior Cycle provides some insight into how wellbeing is taking shape in post-primary education. The findings indicate an overall positive response from teachers involved in delivering the wellbeing programme, however the study highlights that teachers continue to teach their subject areas without consideration of the key skill of wellbeing at junior cycle or the wellbeing indicators, which are a feature of the junior cycle wellbeing guidelines. The study also recommended that time and support to enable collaboration amongst teachers should be provided, given that collaborative practice is key to the successful implementation of wellbeing (Ryan 2018). It is important that further research is undertaken on the implementation of wellbeing at Junior Cycle, and that the findings and recommendations inform future developments in the primary school sector. Otherwise, pupils' wellbeing experience will be rooted in learning about wellbeing and wellbeing may become an umbrella term within which subject areas exist without real content or meaning. There is also the scope to bring wellbeing into the curriculum at Senior Cycle to ensure students leave their post-primary schooling with age-appropriate refined skills to support their health and wellbeing.

Fattore, Mason and Watson (2007) consider that wellbeing in schools is often viewed from a deficit perspective and interventions are implemented in a spurious manner in an attempt to fix a perceived problem. Given that wellbeing has weaved its way into the primary school curriculum space in a somewhat ad-hoc manner, it can certainly be argued that a plethora of wellbeing programmes and initiatives, aimed at enhancing the wellbeing of children have made their way into schools. Some examples include Mindfulness practices, Friends for Life, Zippy's Friends, MindUP, and Weaving Wellbeing. Indeed these programmes, while loosely based on curriculum, and in particular the objectives of SPHE strand and strand units, become a replacement for curriculum. The subject area of SPHE is completely overwhelmed by the number of programmes and resources that are available to schools to support its implementation. This takes teachers further and further away from the objectives of the curriculum and leads to unnecessary feelings of confusion and complication regarding subject implementation. Ensuring that the content of the wellbeing area, and the related subject areas is detailed and comprehensive enough so that it does not become replaced by commercial programmes is a key consideration in curriculum reform. Creating choice should not come at a price of fragmentation and incoherence!

The recently published Wellbeing Policy Statement and Framework for Practice (DES 2018), which will influence wellbeing developments in the primary sector will need to be considered in light of the ongoing work of the NCCA on the redevelopment of the primary school curriculum. The current timeline for developments proposes that following a consultation process, the NCCA will develop a specification for each curriculum area and this is due for completion by summer 2024 . In terms of the wellbeing policy statement, schools have until 2023 to engage in a whole-school self-evaluation process of wellbeing through the four key areas of Culture and Environment, Curriculum, Policy and Planning and Relationships and Partnerships. This timeline has been impacted by the Coronavirus pandemic and may need to be extended. In order to avoid further confusion for schools and teachers about an already 'wicked' problem, it is important that there is a clear alignment between the DES wellbeing policy and the ongoing work of the NCCA. The work undertaken by schools in the area of wellbeing in the coming years should provide a strong foundation upon which schools can build and develop meaningful wellbeing practices. 
Similar to the multiple definitions of wellbeing, word associations related to wellbeing or wellbeing subsets including emotional wellbeing, social wellbeing, psychological wellbeing and mental health and wellbeing complicate the area and add to its multidimensional nature (Svane et al. 2019). The My World Survey highlights that the number one health issue for young people is their mental health (Dooley and Fitzgerald 2012). This is the component of wellbeing teachers feel least qualified to address. While it is important that mental health does not overshadow the other components of wellbeing in the school setting, planning for wellbeing must pay particular attention to this area and ensure school personnel feel competent in supporting children on a day-to-day basis in schools and have access to knowledge on pathways and routes of referral for pupils with particular mental health needs. Furthermore, the development of an interdisciplinary conceptual framework for wellbeing in education, which takes account of its multidimensional nature, is important and necessary to conceptualise wellbeing within an educational realm and ensure that the multiplicity of factors that influence pupil wellbeing are considered.

Schools play a central role in promoting the moral, social, spiritual and personal development of pupils. There is no doubt that wellbeing that incorporates learning about and for wellbeing should feature as a core component of future curriculum iterations. But investment in terms of adequate time for the area, adequate research in terms of what the curriculum component should comprise and adequate support and professional development for teachers are required to enable success of our educational wellbeing initiatives.

The review and redevelopment of the primary school curriculum that is underway by the NCCA is a very exciting time for primary education. The potential to include wellbeing as an area of learning across primary schools reflects what teachers value in schools and is important for the development of our young people and our school communities. Making it a meaningful process for all involves making our wellbeing policy meaningful!

\section{Conflict of interest statement}

The authors declared no potential conflicts of interest with respect to the research, authorship, and/or publication of this article.

\section{Funding Statement}

This research was not supported by any grants or other funding.

\section{Authors}

Margaret Nohilly

Mary Immaculate College, Ireland

Margaret.Nohilly@mic.ul.ie

Fionnuala Tynan

Mary Immaculate College, Ireland

\section{Authors' Contributions}

Both M.N. and F.T. conceptualised and wrote the article and contributed to the final manuscript.

\section{Publishing Timeline}

Received 10 August 2021

Revised version received 20 December 2021

Accepted 20 December 2021

Published 31 January 2022 


\section{References}

Bache, I., L. Reardon, and P. Anand. 2015. "Wellbeing as a wicked problem: Negotiating the arguments for the role of government". Journal of Happiness Studies, 17(3), 893-912.

Beck, J. 2012. "Reinstating knowledge: diagnoses and prescriptions for England's curriculum ills". International Studies in Sociology of Education, 22(1), 1-18.

Blum, R.W., \& Libbey, H.P. (2004). "School connectedness - Strengthening the health and education outcomes for teenagers". Journal of School Health, 74(4), 229-299.

Department of Education \& Department of Health. 2000. Our Children, Their Lives. Accessed January 9, 2019. https://www.dcya.gov.ie/documents/Aboutus/stratfullenglishversion.pdf

Department of Children \& Youth Affairs. 2014. Better Outcomes, Brighter Futures; The National Policy Framework for Children and Young People. Accessed April 4, 2019. https://www.dcya.gov.ie/documents/cypp_framework/BetterOutcomesBetterFutureReport.pdf.

Department of Education \& Skills and Department of Health \& Children. 2013. Well-Being in Post-Primary Schools: Guidelines for Mental Health Promotion and Suicide Prevention. Accessed April 8, 2019. https://www.education.ie/en/Publications/EducationReports/Well_Being_PP_Schools_Guidelines.pdf

Department of Education \& Skills and Department of Health \& Children. 2015. Well-Being Primary Schools: Guidelines for Mental Health Promotion. Accessed April 8, 2019.

https://www.education.ie/en/Publications/Education-Reports/Well-Being-in-Primary-SchoolsGuidelines-for-Mental-Health-Promotion.pdf

Department of Health. 2013. Healthy Ireland, A Framework for Improved Health and Wellbeing, 2013-2025. Accessed April 7, 2019. http://health.gov.ie/wpcontent/uploads/2014/03/HealthyIrelandBrochureWA2.pdf.

Department of Education \& Skills. 2019. Wellbeing Policy Statement and Framework for Practice 2018-2023. Accessed April 19, 2019. https://www.education.ie/en/Publications/Policy-Reports/wellbeing-policystatement-and-framework-for-practice-2018\%E2\%80\%932023.pdf

Deschesnes, M., N. Drouin, C. Tessier, and Y. Couturier. 2014. "Schools' capacity to absorb a healthy school approach into their operations, insights from a realist evaluation". Health Education, 114(3), 208-224.

Dessel, A. 2010. "Prejudice in Schools: Promotion of an Inclusive Culture and Climate". Education and Urban Society, 42, 407-429. 10.1177/0013124510361852.

Dodge, R., A. Daly, J. Huyton, and L. Sanders. 2012. “The challenge of defining wellbeing”. International Journal of Wellbeing, 2(3), 222-235.

Dooley, B., C. O'Connor, A. Fitzgerald and A. O' Reilly. 2019 My world survey 2: National study of youth mental health in Ireland. Accessed July 20, 2020. http://www.myworldsurvey.ie/full-report

Dooley, B., and A. Fitzgerald. (2012) My World Survey: National Survey of Youth Mental Health in Ireland. Accessed July 6, 2020.

http://researchrepository.ucd.ie/bitstream/handle/10197/4286/My_World_Survey_2012_Online(4).pdf ?sequence $=1$

Fattore, T., and E. Mason. 2009. "When children are asked about their well-being: Towards a framework for guiding policy". Child Indicators Research, 2, 57-77.

Gillet-Swan, J. and J. Sargeant. 2015. “Wellbeing as a process of accrual: Beyond subjectivity and beyond the moment". Social Indicators Research, 121(1), 135-148.

Government of Ireland. 1999a. Primary School Curriculum Introduction. Dublin, Stationery Office.

Government of Ireland. 1999b. Social Personal \& Health Education Curriculum. Dublin, Stationery Office.

Jamal F., A. Fletcher, A. Harden, H. Wells, J. Thomas, and C. Bonell. 2013. The school environment and student health: a systematic review and meta-ethnography of qualitative research. BMC Public Health, 13(1), 798.

Leahy, D. and V. Simovska. 2017. "Critical perspectives on health and wellbeing education in schools", Health Education, 117(5), 430-433. 
Lomas T., J. Medina, I. Ivtzan, S. Rupprecht and F. Eiroa-Orosa. 2016. "The impact of mindfulness on the wellbeing and performance of educators: A systematic review of the empirical literature". In Teaching and Teacher Education. Elsevier, pp. 132-141.

National Council for Curriculum and Assessment. 2009. Aistear Framework. Accessed April 19, 2019. http://www.ncca.ie/en/Curriculum_and_Assessment/Early_Childhood_and_Primary_Education/Earl y_Childhood_Education/Framework_for_early_learning/.

National Council for Curriculum and Assessment. 2017. Junior Cycle Wellbeing Guidelines. Accessed April 22, 2019.

http://www.juniorcycle.ie/NCCA_JuniorCycle/media/NCCA/Curriculum/Wellbeing/WellbeingGuidelines-for-Junior-Cycle.pdf

National Council for Curriculum and Assessment. 2017. Junior Cycle Wellbeing Guidelines. Accessed March 24, 2021. https://ncca.ie/media/4940/updated_guidelines_2021.pdf

National Council for Curriculum and Assessment. 2020. Draft Primary Curriculum Framework for Consultation. Retrieved on 02.03.2020 from: https://ncca.ie/media/4456/ncca-primary-curriculumframework-2020.pdf

Nohilly M., and F. Tynan. 2019 "Bridging the gap between the language of policy and the culture of school". International Journal of Educational Policy and Leadership, 15(12), 1-16. https://doi.org/10.22230/ijepl.2019v15n12a886

O' Brien M., and A. O' Shea .2017. A Human Development (PSP) Framework for Orienting Education and Schools in the Space of Wellbeing. Accessed December 20, 2021. https://ncca.ie/media/2488/a-humandevelopment-framework-psp.pdf

O' Toole, C. 2017. "Towards dynamic and interdisciplinary frameworks for school-based mental health promotion". Health Education, 117(5), 452-468.

Ryan, S. 2018. An exploratory study of the impact of the involvement of teachers of SPHE, CSPE and PE in the new Wellbeing programme at Junior Cycle. A teaching perspective. Unpublished Masters Thesis submitted to the University of Limerick. October 2018.

Scottish Government. 2018. Understanding Wellbeing. Accessed May 21, 2019. https://www.gov.scot/binaries/content/documents/govscot/publications/advice-andguidance/2016/03/getting-right-child-understanding-wellbeing-leaflet/documents/getting-right-childunderstanding-wellbeing-considering-quality-children-young-peoples-lives/getting-right-childunderstanding-wellbeing-considering-quality-children-young-peopleslives/govscot\%3Adocument/00498280.pdf

Smyth E. 2012. The Primary Classroom: Insights from the Growing Up in Ireland Study. Accessed July 15, 2020. https://www.esri.ie/publications/wellbeing-andschool-experiences-among-9-and-13-year-oldsinsights-from-the-growing-up-in-ireland-study/

Soutter, A.K., B. O' Steen, and Gilmore A. 2014. "The student well-being model: a conceptual framework for the development of student well-being indicators". International Journal of Adolescence and Youth, 19(4), 496-520.

Svane, D., E. Neus, and M. Carter. 2019 "Wicked wellbeing: Examining the disconnect between the rhetoric and reality of wellbeing interventions in schools". Australian Journal of Education, 63(2), 209231.

Thorburn, M. 2015. "Theoretical constructs of well-being and their implications for education". British Educational Research Journal, 41(4), 650-665.

Turner, K., and M. Theilking. 2019. “Teacher Wellbeing: Its Effects on Teaching Practice and Student Learning Issues". Educational Research Journal, 29(3), 938-960. http://www.iier.waier.org.au/iier29/turner2-abs.html.

Tynan F., and M. Nohilly. 2018. Wellbeing in School's Everyday (WISE) A whole-school approach to the practical implementation of wellbeing. Curriculum Development Unit, Mary Immaculate College.

Young, M. 2013. "Overcoming the crisis in curriculum theory: a knowledge-based approach". Journal of Curriculum Studies, 45(2), 101-118.

Weare, K. 2000. Promoting mental, emotional, and social health: A whole-school approach. London: Routledge. 